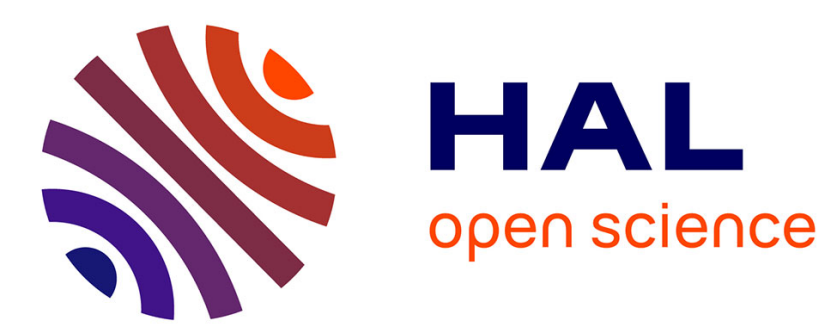

\title{
Implementation of surface tension force in fluid flow during reactive rotational molding
}

A. Hamidi, Sofiane Khelladi, A. Illoul, Mohammadali Shirinbayan, Farid Bakir, Abbas Tcharkhtchi

\section{- To cite this version:}

A. Hamidi, Sofiane Khelladi, A. Illoul, Mohammadali Shirinbayan, Farid Bakir, et al.. Implementation of surface tension force in fluid flow during reactive rotational molding. International Journal of Material Forming, 2015, 9 (2), pp.131-148. 10.1007/s12289-015-1217-z . hal-02446893

\section{HAL Id: hal-02446893 \\ https://hal.science/hal-02446893}

Submitted on 21 Jan 2020

HAL is a multi-disciplinary open access archive for the deposit and dissemination of scientific research documents, whether they are published or not. The documents may come from teaching and research institutions in France or abroad, or from public or private research centers.
L'archive ouverte pluridisciplinaire HAL, est destinée au dépôt et à la diffusion de documents scientifiques de niveau recherche, publiés ou non, émanant des établissements d'enseignement et de recherche français ou étrangers, des laboratoires publics ou privés. 


\title{
Implementation of surface tension force in fluid flow during reactive rotational molding
}

\author{
A. Hamidi • S. Khelladi • A. illoul • M. Shirinbayan • \\ F. Bakir • A. Tcharkhtchi
}

\begin{abstract}
During Reactive Rotational Molding (RRM), it is very important to predict the fluid flow in order to obtain the piece with homogeneous shape and high quality. This prediction may be possible by simulation the fluid flow during rotational molding. In this study we have used a mixture of isocyanate and polyol as reactive system. The kinetic rheological behaviors of thermoset polyurethane are investigated in anisothermal conditions. Thanks to these, rheokinetik model of polyurethane was identified. Then, to simulate the RRM, we have applied Smoothed Particles Hydrodynamics (SPH) method which is suited method to simulate the fluid flow with free surface such as occurs at RRM. Modelling and simulating reactive system flow depend on different parameters; one of them is the surface tension of reactive fluid. To implement force tension surface, the interface between polymer and air is dynamically tracked by finding the particles on this border. First, the boundary particles are detected by free-surface detection algorithm developed by Barecasco, Terissa and NAA $[1,2]$ in two and three dimension. Then, analytical and geometrical algorithms have been used for interface reconstructions. The aim of this work is the implementation of surface tension force in the SPH solver applied to RRM. To illustrate that, we used novel and simple geometric algorithm fitting circle and fitting sphere, in two and three dimensional configurations, respectively. The model has been validated using a well-known dam break test case which covered the experimental data.
\end{abstract}

A. Hamidi $(\bowtie) \cdot$ A. illoul $\cdot$ M. Shirinbayan $\cdot$ A. Tcharkhtchi

Art et Métiers ParisTech, UMR 8006, PIMM,

151 bd de l'hôpital, 75013 Paris, France

e-mail: abdelmoumen.hamidi@ensam.au

S. Khelladi $\cdot$ F. Bakir

DynFluid laboratory, Art et Métiers ParisTech,

151 Bd de l'hôpital, 75013 Paris, France
Keywords Reactive rotational molding · Simulation Smoothed particle hydrodynamics · Surface tension · Polyurethane $\cdot$ Interpolation

\section{Introduction}

Rotational molding is a four-stage, high-temperature (150$300{ }^{\circ} \mathrm{C}$; depend on the polymer), low-pressure, plastic molding process that uses heat and bi-axial rotation to produce hollow, one-piece parts [3, 4].

Rotational molding is known as an economically and environmentally viable method for manufacturing of polymers. The molds of this process are less expensive because it's not necessary to make them with high performance materials. In absence of pressure, there is no residual stress in final parts. In this manufactory method, the quantities of wasted materials are low. However, this process takes long times, about 20 $40 \mathrm{~min}$; so this technique is generally used for small series of industrial parts. The limited number of polymers only thermoplastics that can be used for rotational molding is also considered as another disadvantage of this process. The various inconvenient of conventional rotomolding have favored the emergence of RRM where the polymer synthesis and piece shaping are simultaneously carried which allows to reduce cycle time and expand the range of materials such as thermoset [5]. Different parameters involved during RRM such as rate of chemical rate, viscosity variations and fluid flow. These phenomena are complex and require detailed study to modeling the process.

Previous work permits to simulate thermoset polyurethane in two and three dimensional configuration using SPH [6-8]. In order to complete our SPH solver because we have observed as it works can generate roughness or particle 
agglomerates on the internal surface (Fig. 1). This phenomenon affects the flow of material and its adhesion process that can be slowed and even stopped. It will also consider this problem for example by integrating new model or criterion then seeing if taking into account the viscoelasticity of the material and / or surface tension, this phenomenon can be reduced. In this study, the surface tension force will be integrated in SPH solver in order to simulate the polymer flow during RRM.

Surface tension has a significant role in many free surface flows, such as occurs to RRM. It is necessary to include surface tension into the SPH solver to make realistic simulations. Many models have been integrated in SPH method developed to study fluid flows with surface tension. Since surface tension is defined as a property of liquids arising from unbalanced molecular cohesive forces at or near the surface, Nugent and Posch [9] applied the SPH method to formation of a liquid drop from an initial regular shape. The cohesive pressure in the Van der waals (Vdw) equation of state is similar to the unbalanced molecular cohesive forces that cohesive pressure works as surface tension.

Then, the Vdw model is applied to simulate the oscillation of deformed drop without tensile instability [10,11]. After that, Tartakovsky and Meakin $[12,13]$ introduced new term in the continuity equation based on interaction particle force to simulate the effect of the tension surface. Another treatment of surface tension in SPH method based on the continuum surface force (CSF) model developed by Brackbill et al. [14], where the interface curvature is calculated through a color function (Morris [15]). The main disadvantage of this method was the estimation error of surface tension for large curvature because the second derivative of a color function is sensitive to particle disorder.

Zhang [16] proposed a new surface tension method where free surface boundary can be dynamically tracked by using

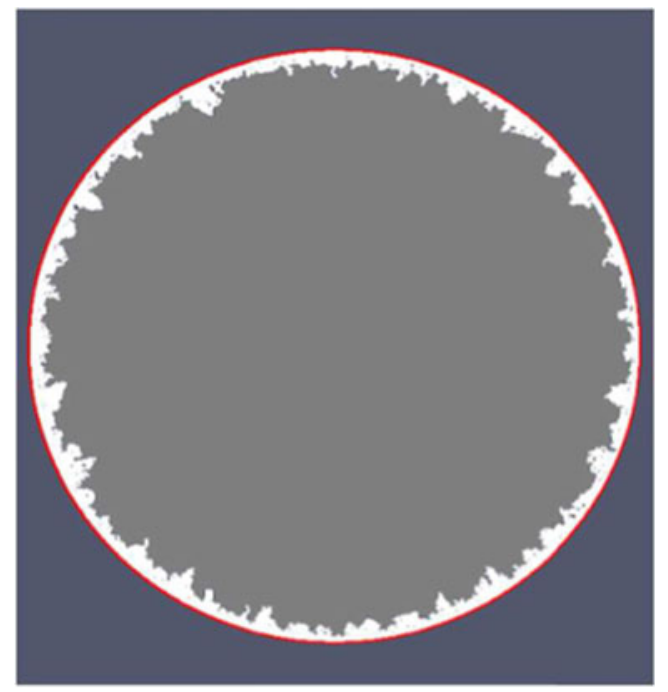

Fig. 1 Formation of particle agglomerates on the internal surface of part geometric algorithms $[17,18]$. But this method is quite difficult to implement it and particularly in three dimensions. However, Marrone [19] presented a new method based on the properties renormalization matrix, defined by Randles and Libersky [20]. Recently, Barecasco and Al [1, 2] developed new method which the domain is represented by overlapping of spheres where each sphere represents a SPH particle centered at the particle's position. If the sphere of a SPH particle is not completely covered by the spheres of its neighbors, the SPH particle is a boundary particle. Otherwise, it is an inner particle.

The objective of this work is to develop a numerical method for simulating reactive fluid flow during RRM taking into account the free surface tension and meeting the following requirements: implement simple algorithm to detect the interface air-polymer using algorithm developed by barecasco and Al., construct by geometrical algorithm like fitting circle (2D) and sphere (3D), finally, show the capability to implement surface tension force in SPH solver and compare our method with other method that were used in the literature.

\section{Method and material}

The fluid flows during RRM of liquid polymer will be simulated by SPH method. The reactive fluid is modeled as an incompressible viscous Newtonian fluid because the viscosity remains low until gelation and the shear force is neglected for the reason that the mold rotates very slowly (1 to $10 \mathrm{rpm}$ ). The surface tension force is only applied for boundary particles.

To perform this study, we use the reactive fluid composed by isocyanate and a polyol with functionality more than two in order to synthetize thermoset polyurethane.

\section{Kinetic and Rheokinetik modelling}

\section{Kinetic modelling}

The knowledge of kinetic parameters of reactive thermoset polyurethane is essential on the design and processing of polymer technology. Kinetic prediction of the cure pattern in dynamic conditions is very interesting. Polyurethane thermoset studied here, is produced by the polyaddition of a polyisocyanate and a Polyol. The mechanism of the polymerization of polyurethane have been investigated in the literature, mostly to the classical second order-reaction [21, 22]. In this study, we use kamal and serror's model [23] to describe the kinetic of this system:

$\frac{d \alpha}{d t}=\left(k_{1}+k_{2} \alpha^{n}\right)(1-\alpha)^{m}$ 
Where $\alpha, \mathrm{k}_{1}, \mathrm{k}_{2}, \mathrm{n}$ and $\mathrm{m}$ represent the conversion, reaction constant and order reactions respectively.

\section{Rheokinetik modelling}

As a result of the reaction between Polyol and Isocyante, the reactive system first increase in viscosity and eventually crosslinks and become set, reaching gel point, and as a result it can no longer flow or dissolve. Therefore, it is important to understand the rheological behavior of the thermosetting resin in order to optimize the operating conditions for a reactive polymer process that includes both reaction and viscous. In order to model a reactive polymer process, a viscosity function that can express the relationship between viscosity and temperature and conversion is necessary. In this study, we used Castro and Macosko model [24] which give relationship between the viscosity and conversion, as:

$\eta=\eta_{0}\left(\frac{\alpha_{\mathrm{gel}}}{\alpha_{\mathrm{gel}}-\alpha}\right)^{\mathrm{a}+\mathrm{b} \alpha}$

Where $\alpha_{\text {ge1 }}$ represents the conversion at gelation point, and $\eta_{0}$, $\mathrm{a}$ and $\mathrm{b}$ are empirical constants.

\section{Experimental results and discussions}

Thermal properties

The Differential Scanning Calorimetry is widely used to analyse the cure kinetics of thermosets. Linear heating runs are carried out in a TA instruments DSC Q10 thermal analyser.
Samples were prepared with approximately $10 \mathrm{mg}$ of the mixture (polyol and isocyanate) in hermetic aluminium capsules. During the study the samples are heated from -20 to $220^{\circ} \mathrm{C}$ at different heating rates $2,5,7,10$ and $15^{\circ} \mathrm{C} / \mathrm{min}$. The DSC test results for dynamic cure at different heating rates are represented in thermograms such as the one illustrated in Fig. 2. The shape of the thermograms shows two exothermic peaks major peak around $140{ }^{\circ} \mathrm{C}$ correspond to the crosslinking of polyurethane and the second peak represents secondary reaction due to the consumption of NCO by side reactions as formation of allophonates [25].

We use Datapaq telemetry tracker to show the real change of temperature during RRM. Figure 3 depicts the evolution of temperature of the reactive system and the oven. The ramp of temperature observed by these measurements corresponds to $7^{\circ} \mathrm{C} / \mathrm{min}$. In the rest of our work, we determine the laws governing the change of kinetic and rheokinetik at this heating rate.

In this section, the kinetics is studied by following the consumption of Isocyanate function. Conversion is plotted as a function of temperature at $7{ }^{\circ} \mathrm{C} / \mathrm{min}$. An attempt was made to fit this experimental data to the kamal and Serror model. Figure 4 shows the experimental results and the numerical results for the reaction between isocyantes and polyols. A reasonable fit of the reaction profile could be achieved with kamal and serror model.

\section{Rheological measurements}

Rheological experiments were performed using an Ares Rheometer from TA Instruments equipped with $25 \mathrm{~mm}$ diameter parallel plates to assess the rheological properties of the polyurethane reacting system, called dynamic temperature ramp in order to determine the system's viscosity at $7^{\circ} \mathrm{C} / \mathrm{min}$.
Fig. 2 Evolution of the heat flux with temperature for the nonisothermal scans

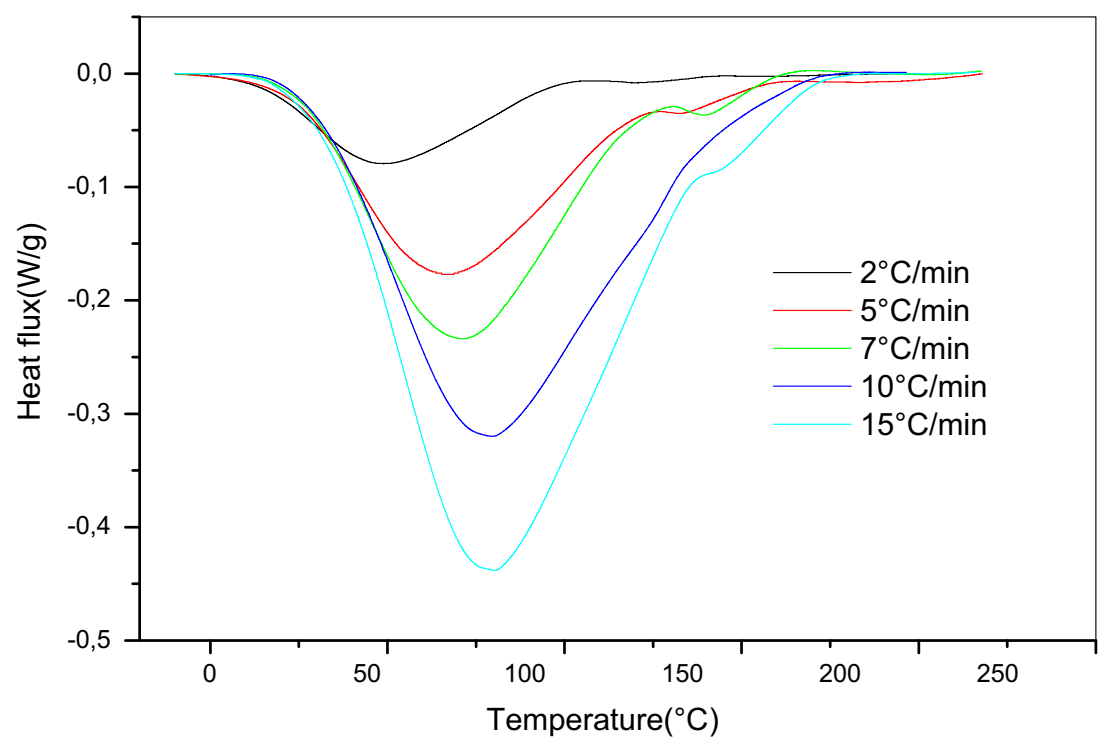


Fig. 3 Temperature curves during RRM of Polyol and isocyantes system

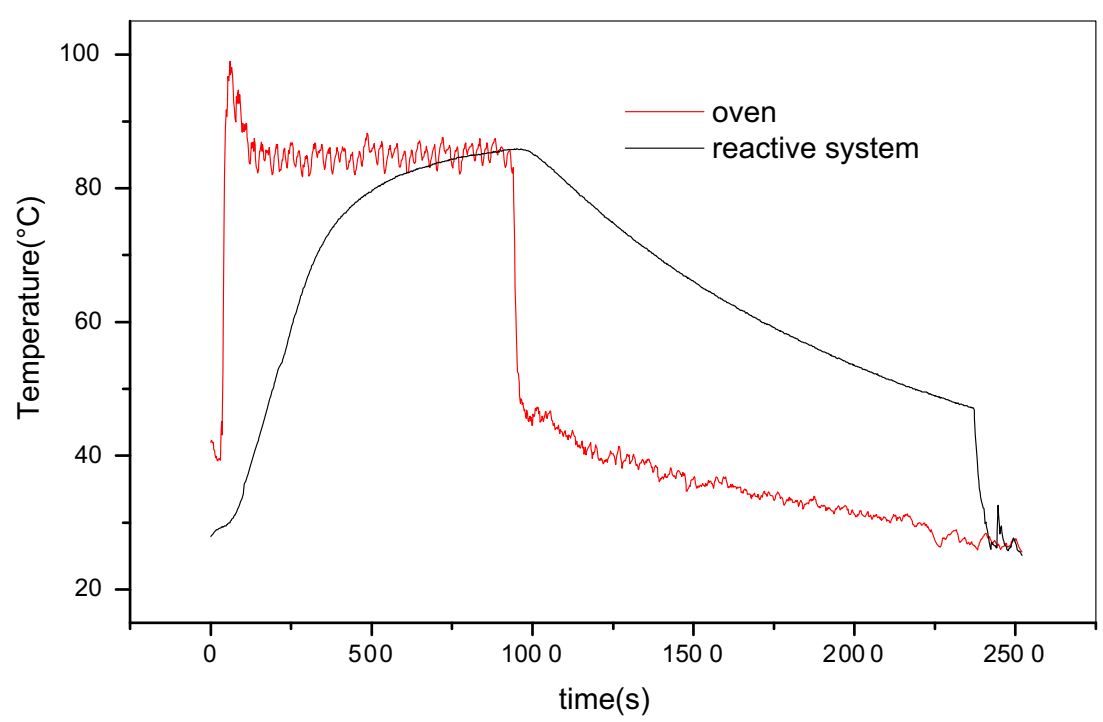

The material's rheological measurements are taken each $5 \mathrm{~s}$ and the gap between parallel plates is between 0.5 and $1 \mathrm{~mm}$ wide. A thermoset reaches its gel point when the elastic modulus equals the loss modulus. The Fig. 5 depicted an example of the evolution of the change of storage modulus (G') and loss modulus (G”).

The Table 1 presents the material's gel temperature, time and conversion obtained at $7{ }^{\circ} \mathrm{C} / \mathrm{min}$.

In order to correlate the kinetic model, $\alpha$ and the experimental rheological results, $\eta(t, T)$, the Macko-Castro chemorheological model presented in Eq. (1) is used. As shown in Fig. 6, the model describes successfully the evolution of viscosity. Parameters are determined through regression based on error minimization $\left(\eta_{0}=0.94, a=-3.27\right.$ et $b=$ $-2.41)$. There is reasonably good between numerical and experimental results. In our simulations, we use this rheokinetik model to describe the evolution of viscosity of our material during RRM. In our simulations, we use this model with to describe the evolution of reactive system's viscosity during the process.

\section{Numerical model: RRM simulations}

The study of fluid flow is described by the Navier-Stokes equations that reflect the mass conservations, momentum and total energy. A state equation is combined to these equations in order to describe the thermodynamic behavior of fluid. In Lagrangian formalism these equation can be written as [26]:

$\nabla \vec{v}=0$
Fig. 4 Simulated (-) and experimental conversion (-) data versus temperature for $7^{\circ} \mathrm{C} / \mathrm{min}$

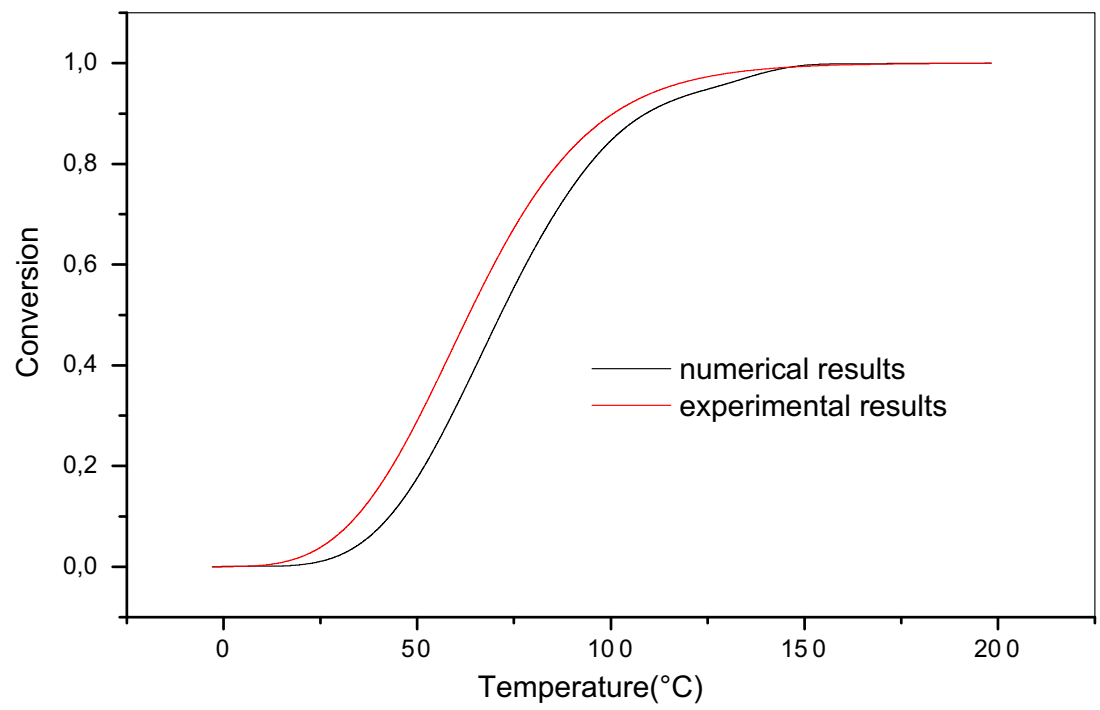


Fig. 5 The result of temperature sweep experiment at the heating rate of $7{ }^{\circ} \mathrm{C} / \mathrm{min}$ on the change of storage modulus (G') $\frac{\partial \vec{v}}{\partial t}=g+\frac{1}{\rho} \nabla \cdot \tau-\frac{1}{\rho} \nabla P+\frac{1}{\rho} \vec{f}$

Where: $g, P, \mathrm{v}$ and $\rho$ represent the gravitational acceleration, pressure, the velocity vector and density, respectively. $\vec{f}$ denotes the surface force which acts only at the interface only.

Among the methods that allow us to simulate the free-surface flow, there are meshless methods in particular Smoothed Particle Hydrodynamics method [27, 28]. The principle of the SPH method is based on field reconstruction from a cloud of discrete and disordered points.

This approximation in discrete notation permit to find the value of the scalar field $A(r)$ at any point, at a particle $a$ the following equation is used:

$A(r)=\sum_{b} \frac{m_{b}}{\rho_{b}} A_{b} W_{a b}$

Where: the summation is over all the particles within the region of compact support of kernel function. $\mathrm{a}, \mathrm{b}$ represent the SPH particles; $m_{b}$ and $\rho_{b}$ are mass and density of particle $b$, respectively. $W_{a b}$ is weight or kernel function which has compact support.

Table 1 Reactive system gel: temperature, time and conversion

\begin{tabular}{lc}
\hline Gel point & $7^{\circ} \mathrm{C} / \mathrm{min}$ \\
\hline time $(\mathrm{s})$ & 573 \\
Temperature $\left({ }^{\circ} \mathrm{C}\right)$ & 75.27 \\
Conversion $\left(\alpha_{\text {gel }}\right)$ & 0.68 \\
\hline
\end{tabular}

In $\mathrm{SPH}$, the fundamental principle is to approximate any function $A(r)$ by:

$A(r)=\int A\left(r^{\prime}\right) W\left(r-r^{\prime}, h\right) d r^{\prime}$

Where: $h$ is smoothing length;

In present simulation, the cubic spline kernel has been used:

$W(r, h)=\alpha_{D}\left\{\begin{array}{lr}1-\frac{3}{2} q^{2}+\frac{3}{4} q^{3} & 0 \leq q \leq 1 \\ \frac{1}{4}(2-q)^{3} & 1 \leq q \leq 2 \\ 0 & q \geq 2\end{array}\right.$

Where $q=r / h, r$ is the distance between particle $a$ and $b$.

$\alpha_{D}$ is $10 /\left(7 \pi h^{2}\right)$ in $2 \mathrm{D}$ and $1 /\left(\pi h^{3}\right)$ in $3 \mathrm{D}$.

The Navier-Stokes equations are discretized by the SPH method for each particle, will be given below, the continuity Eq. (8), momentum Eq. (9) and thermal energy (11).

$\frac{d \rho_{a}}{d t}=\sum_{b} m_{b}\left(\vec{v}_{a}-\vec{v}_{b}\right) \vec{\nabla} W_{a b}$

Where: $\rho_{a}, v_{a}$ are density and velocity of particle $a$, respectively; $m_{b}$ and $v_{b}$ are mass and velocity of particle $b$.

$$
\begin{aligned}
\frac{d \vec{v}_{a}}{d t}= & -\sum m_{b}\left(\frac{P_{a}}{\rho_{a}^{2}}+\frac{P_{b}}{\rho_{b}^{2}}\right) \vec{\nabla} W_{a b} \\
& +\sum \frac{m_{b}\left(\eta_{a}+\eta_{b}\right) v_{a b}}{\rho_{a} \rho_{b}}\left(\frac{1}{r_{a b}} \frac{\partial W_{a b}}{\partial r_{a}}\right)+\vec{g}+\vec{f}
\end{aligned}
$$


Fig. 6 Viscosity change comparison between experimental and macko-castro model (घ numerical results• experimental values)

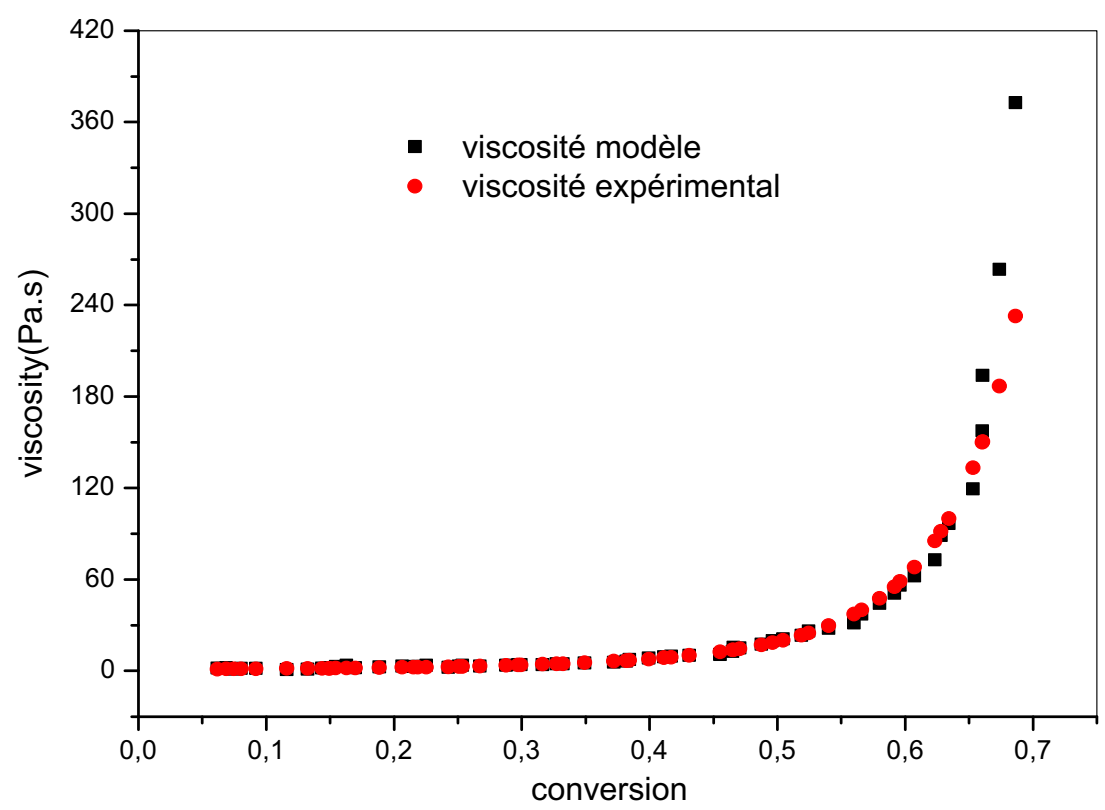

Where: $P_{a}, P_{b}$ and $\eta_{a}, \eta_{b}$ are pressure and viscosity of particles $a$ and $b$, respectively and $\vec{g}$ represents gravity. And $\vec{f}$ :surface tension surface and is given by:

$\vec{f}=\sigma k \vec{n} \delta_{s}$

$\sigma$ : coefficient of surface tension; $k$ : the curvature; $\vec{n}$ : normal vector of surface; $\delta_{s}$ : surface delta function and it's equal to $1 / \varepsilon$ where: $\varepsilon$ represents particle spacing.

$\frac{d E_{a}}{d t}=\sum_{b} \frac{4 m_{b}}{\rho_{a} \rho_{b}} \frac{k_{a} k_{b}}{k_{a}+k_{b}} T_{a b} \frac{r_{a b} \cdot \nabla_{a} W_{a b}}{r_{a b}^{2}+\varepsilon^{2}}$

$k_{a}$ and $k_{b}$ are respectively the conductivity of particle $a$ and $b, T_{a b}$ is the difference between temperatures of particles $a$ and $b$. This equation involves an explicit conductivity which can be variable. This allows simulating heat transfer of multiple materials with different conductivities.

To describe the thermodynamic behaviour of the fluid, we have associated to this system an equation of state which shows the variation pressure $\mathrm{P}$ in function of density $\rho$.

$P=P_{0}\left[\left(\frac{\rho}{\rho_{0}}\right)^{\gamma}-1\right]$

Where: $P_{0}$ is the magnitude of the pressure and $\rho_{0}$ is the reference density. $P_{0}$ is given by:

$P_{0}=\frac{c^{2} \rho_{0}}{\gamma}$

With $c_{S}$ is speed sound at the reference density and $\gamma$ is a problem dependent parameter.

\section{Treatment of boundary conditions}

This section summarizes our wall boundary conditions. several techniques are utilized in the literature, to treat wall boundaries, for example, by using mirror particles [28], dummy particles [20] and repulsive force [27]. A widely used approach, proposed by Monaghan, is the repulsive force method where the wall is described by particles which exert a repulsive short-range force similar to a Leonard-Jones potential force on fluid particles. This last force has been used in our simulations. Moreover, with repulsive forces, only one layer of particles is placed on the wall with identical mass and density to inner particles. A type of Lennard-Jones force is used as the repulsive force, $\mathrm{PB}_{\mathrm{ab}}$ :

$P B_{a b}= \begin{cases}D\left[\left(\frac{r_{0}}{r_{a b}}\right)^{n_{1}}-\left(\frac{r_{0}}{r_{a b}}\right)^{n_{2}}\right] \frac{x_{a b}}{r_{a b}^{2}} & \text { if }\left(\frac{r_{0}}{r_{a b}}\right) \leq 1 \\ 0 & \text { if }\left(\frac{r_{0}}{r_{a b}}\right)>1\end{cases}$

The values of $n_{1}$ and $n_{2}$ are usually taken 12 and 4 respectively, $D$ should be chosen to be in same scale as the square of largest velocity and $r_{0}$ is distance from which fluid particles interact with solid particles.

Unfortunately, the boundary conditions adopted in our study produce no-slip conditions. In this configuration, fluid particles cannot adhere to the boundary particles (except for very low velocities). To simulate the adhesion of RRM on the mold surface, Riviere [8] developed a model to fix the fluid particles when they reached a certain viscosity and they are exposed to a certain distance from the mold (or fixed material) during a certain time. Figure 7 illustrates a schematic 
Fig. 7 Adhesion schematic

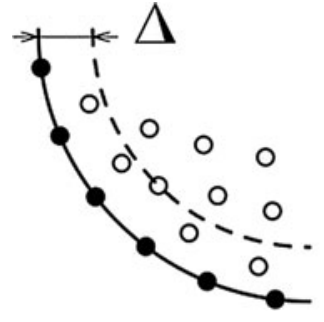

- boundary particles (mold or adhered polymer)

○ fluid particles (polymer)

$\triangle$ adhesion length representation of fluid particles and the boundary particles (mold or adhered polymer). The adhesion length is distance from boundary to situation which fluid particles can be adhered. For fixing a fluid particle, three conditions must be verified:

- The effective particle viscosity $\eta_{a}$ must be higher than adhesion viscosity, $\eta_{\text {adhe }}$ (The viscosity value which polymer starts to adhere on the mold surface).

- The distance of fluid particle and boundary $r_{a b}$ must be lower than adhesion length $\Delta$.

- The exposure time $t_{\text {expo }}$ of a fluid particle must be higher than adhesion time $t_{\text {adhe }}$. Where count-down is engaged when $r_{a b} \leq \Delta$.

$\eta_{\text {adhe }}$ and $t_{\text {adhe }}$ are physical parameters linked to surface roughness and rotation speed of the mold. On the other hand, $\Delta$ is a numerical parameter and is chosen to be in the same scale than the smoothing length. When polymer gelation occurs, the material becomes stiff and there is no more flow. The plastic part can be removed from the mold. To simulate gelation phenomena, the remaining fluid particles must be fixed. When the fluid particles viscosities $\eta_{a}$ reach a viscosity close to the gel point $\eta_{g e l}$, they become fixed, even if they do not satisfied the three above mentioned criteria. At this step, the simulation process is completed.

In this implementation, sums must check for all particles in the fluid domain if they are within the kernel. To make a faster calculation, we used a linked-list grid method to reduce the calculation amount per particle. In this study, the numerical integration scheme is explicit scheme called Newmark algorithm [29] integrator to advance in time, the variables are calculated according to:

$v_{i}^{n+1}=v_{i}^{n}+\Delta t\left((1-\alpha) a_{i}^{n}+\alpha a_{i}^{n+1}\right)$

$x_{i}^{n+1}=x_{i}^{n}+\Delta t v_{i}^{n}+\Delta t^{2}\left((0.5-\beta) a_{i}^{n}+\beta a_{i}^{n+1}\right)$

Where $x$ and $v$ represent position and velocity, respectively. Moreover $\alpha=0.5$ and $\beta=0.25$. Temperature and density are updated according to (12) and time step $\Delta t$ depends on the Courant Friedrichs Lewy (CFL) condition [30].

On parallel applied optimizations, implementation part is completed with an approach into parallel programming using Open Multi-Processing to achieve shorter simulation runtimes.

\section{Treatment of the surface tension}

Generally, in the case of biphasic systems (polymer-air) surface tension forces are caused by the unbalanced molecular dynamic forces at the free surface, in the region between two different immiscible phases. In these regions the polymer molecules, represented by our particles, are forced to shift in the direction of surface normal towards the liquid itself causing a minimization of liquid's curvature at the surface.

The surface tension force is only present in the border polymer-air, it is necessary to detect dynamically all the particles belonging to the interface. Then, we reconstruct the border air-polymer by Lagrangian interpolation or by circle approximation in 2D and the reconstruction of surface using fitting sphere in three dimensional configurations.

Tracking free surface boundary polymer-air

The interface is dynamically detected by finding all boundary particles using a simple free-surface detection method in two and three-dimensional algorithm developed by Barecasco and Al. In this method, the domain is representing by overlapping of spheres (SPH particle $\mathrm{s}_{\mathrm{i}}$ ), centred at the particle's position $\mathrm{x}_{\mathrm{i}}$ and $r_{i}$. It is then necessary to check if some part of segments of this sphere is or not covered. If there is some piece which isn't covered by the spheres neighbours', it called a boundary particle. Otherwise, it's inner particle. Due to the non-uniform properties of SPH particles, we used a scan cone around the cover vector of the fluid surface for checking any covered particle.

The cover vector can be written as:

$$
b_{i}=\sum_{j=0}^{n} \frac{x_{i}-x_{j}}{\left\|x_{i}-x_{j}\right\|}
$$


We defined an angle $\theta_{\mathrm{i}}$, the angle of cone, in order to test a boundary status of SPH particle.

The particle is considered a boundary particle if:

$\arccos \left(\frac{x_{j}-x_{i} b_{i}}{\left\|x_{j}-x_{i} b_{i}\right\|}\right) \leq \frac{\theta_{i}}{2}$

Reconstruction of the interface in 2D

The curve interface is reconstructed using the boundary particles by two methods: Lagrangian interpolation or fitting circle. Before reconstruction of the curve, the system coordinates are transformed into local coordinate system, in order to guarantee the interface curve will have one-valued in the local coordinate system.

The origin o' in local coordinate system is given by:

$x_{o^{\prime}}=\sum_{j} x_{j} / N_{i}$

$y_{o^{\prime}}=\sum_{j} y_{j} / N_{i}$

Where $\mathrm{j}$ is the particle index of neighbors of boundary particle $i$ and $N_{i}$ is total number of neighbors of boundary particle $\mathrm{i}$.

The relationship between local coordinate (x', y') and coordinate system $(\mathrm{x}, \mathrm{y})$ is given by:

$x=x^{\prime} \cos \alpha-y^{\prime} \sin \alpha$

$y=x^{\prime} \sin \alpha+y^{\prime} \cos \alpha$

Where: $\alpha$ represents the angle between the two axis $\mathrm{x}$ and $\mathrm{x}$ '.

\section{Fitting circle}

First, we used the fitting to reconstruct the boundary curve in the local coordinate system. This method is widely used in metrology and microwave measurement [31-32]. The principle of fitting circle method is to compute an initial guess by averaging all circles that can be built using all triplets of non-aligned points, and then to iteratively reduce the distance between circle and the complete set of points using a minimization method.

A fit measure of the circle with the center being at the point $(a, b)$ and the radius being " $r$ " to the boundary particles points $\mathrm{P}\left(\mathrm{x}_{\mathrm{i}}, \mathrm{y}_{\mathrm{i}}\right)$ is given by summing the squares of distances from these points to the circle. The measure is formalized as follow:

$S(a, b, r)=\sum_{i=1}^{n}\left(r-\sqrt{\left(x_{i}-a\right)^{2}+\left(y_{i}-b\right)^{2}}\right)^{2}$

Where: the index $\mathrm{i}$ indicate a boundary particle.

To determine $a, b$ and $r$ in least square development the values of $a, b$ and $r$ can be determined by differentiating $S(a$, $b, r)$ in relations to circle parameters and forcing the partial derivatives to be zero in order to obtains the values of $a, b$ and $r$. The differentiation of $S(a, b, r)$ are:

$\frac{\partial S}{\partial r}=-2 \sum_{i=1}^{n} \sqrt{\left(x_{i}-a\right)^{2}-\left(y_{i}-b\right)^{2}}+2 n r$

$\frac{\partial S}{\partial a}=2 r \sum_{i=1}^{n} \sqrt{\left(x_{i}-a\right)^{2}-\left(y_{i}-b\right)^{2}}-2 n \bar{x}+2 n a$

$\frac{\partial S}{\partial b}=2 r \sum_{i=1}^{n} \sqrt{\left(x_{i}-a\right)^{2}-\left(y_{i}-b\right)^{2}}-2 n \bar{y}+2 n b$

We note the solving (24) $=0$ for $r$ reaches to:

$r=\sum_{i=1}^{n} \sqrt{\left(x_{i}-a\right)^{2}-\left(y_{i}-b\right)^{2} / n}$

If we obtain a, b by some other method, we can obtain good value of $r$ by using (25).

In this study, we used average of intersections method to fit circle to data points in order to obtain the coordinate of our circle [33]. For this reason, we use geometry properties of circle; in particular the perpendicular bisectors of all chords intersect at the center. For any given triplet of non-aligned points, we determined the intersection $\mathrm{C}_{\mathrm{ijk}}$ of the perpendicular bisectors of side $<\mathrm{Pi}, \mathrm{Pj}>$ (Eq. 27) and the perpendicular bisector $<\mathrm{Pi}, \mathrm{Pj}>$ (Eq. 28).

$\left\{\begin{array}{l}x_{C_{i, j, k}}=\frac{\left(x_{i}+x_{j}\right)+\alpha_{i, j}\left(y_{j}-y_{i}\right)}{2} \\ y_{C_{i, j, k}}=\frac{\left(y_{i}+y_{j}\right)-\alpha_{i, j}\left(x_{j}-x_{i}\right)}{2}\end{array}\right.$

$\left\{\begin{aligned} x_{C_{i, j, k}} & =\frac{\left(x_{j}+x_{k}\right)+\alpha_{k, j}\left(y_{k}-y_{j}\right)}{2} \\ y_{C_{i, j, k}} & =\frac{\left(y_{i}+y_{k}\right)-\alpha_{j, k}\left(x_{k}-x_{j}\right)}{2}\end{aligned}\right.$ 
Solving this set of linear equations reaches to:

$$
\left\{\begin{array}{l}
\alpha_{i, j}=\frac{\left(x_{k}-x_{i}\right)\left(x_{k}-x_{j}\right)+\left(y_{k}-y_{i}\right)\left(y_{k}-y_{j}\right)}{\Delta} \\
\alpha_{j, k}=\frac{\left(x_{k}-x_{i}\right)\left(x_{j}-x_{i}\right)+\left(y_{k}-y_{i}\right)\left(y_{j}-y_{i}\right)}{\Delta}
\end{array}\right.
$$

Where

$$
\Delta=\left(x_{k}-x_{j}\right)\left(y_{j}-x_{i}\right)-\left(x_{j}-x_{i}\right)\left(y_{k}-y_{j}\right)
$$

The coordinates of the circle are:

$$
\left\{\begin{array}{l}
x_{C_{i, j, k}}=\frac{\left(y_{k}-y_{i}\right)\left(x_{i}^{2}+y_{i}^{2}\right)+\left(y_{i}-y_{k}\right)\left(x_{j}^{2}+y_{j}^{2}\right)+\left(y_{j}-y_{i}\right)\left(x_{k}^{2}+y_{k}^{2}\right)}{2 \Delta} \\
y_{C_{i, j, k}}=-\frac{\left(x_{k}-x_{i}\right)\left(x_{i}^{2}+y_{i}^{2}\right)+\left(x_{i}-x_{k}\right)\left(x_{j}^{2}+y_{j}^{2}\right)+\left(x_{j}-x_{i}\right)\left(x_{k}^{2}+y_{k}^{2}\right)}{2 \Delta}
\end{array}\right.
$$

\section{Lagrangian interpolation}

Another method has been used in our study to reconstruct the interface curve by using Lagrangian interpolation. The Lagrange interpolation is formulated as follows:

$P(x)=\sum_{j} P_{j}(x)$

Where:

$P_{j}(x)=y_{j} \prod_{k \neq j} \frac{\left(x-x_{k}\right)}{\left(x_{j}-x_{k}\right)}$

$\mathrm{j}$ is particle index including boundary particle $\mathrm{i}$ and its neighbors on the boundary.The curvature and the normal in local coordinate are obtained as:
$k=\frac{\left|P^{\prime \prime}(x)\right|}{\left[1+P^{\prime 2}(x)\right]^{3 / 2}}$

$\vec{n}^{\prime}=\left\{\begin{array}{c}\left.P^{\prime}(x),-1\right\rangle \text { if } P^{\prime \prime}(x)<0 \\ \left.-P^{\prime}(x),+1\right\rangle \text { if } P^{\prime \prime}(x)>0\end{array}\right.$

The coordinate of normal vector in the original system are given by:

$\vec{n}^{\prime}=\left\{\begin{array}{c}\left.P^{\prime}(x) \cos \alpha+\sin \alpha, P^{\prime}(x) \sin \alpha-\cos \alpha\right\rangle \text { if } P^{\prime \prime}(x)<0 \\ \left\langle-P^{\prime}(x) \cos \alpha-\sin \alpha,-P^{\prime}(x) \sin \alpha+\cos \alpha\right\rangle \text { if } P^{\prime \prime}(x)>0\end{array}\right.$

Reconstruction of the surface in $3 \mathrm{D}$

The boundary surface is reconstructed using fitting sphere method. Before reconstruction of the curve, the system coordinates are transformed into local coordinate system, in order to guarantee the surface curve will have one-valued in the local coordinate system.

The origin o' of the local coordinate system is given by:

$\vec{r}_{o^{\prime}}=\sum_{j} \vec{r}_{j} / N_{i}$

Where $\mathrm{j}$ is the particle index of neighbours of boundary particle $\mathrm{i}$ and $\mathrm{Ni}$ is total number of neighbours of boundary particle $\mathrm{i}$.

The local three-dimensional basis vectors were calculated, by the system of Eq. (38):

$\widehat{Z}_{o^{\prime}}=\frac{\vec{r}-\vec{r}_{o^{\prime}}}{\left\|\vec{r}-\vec{r}_{o^{\prime}}\right\|}, \widehat{X_{o^{\prime}}}=\left\{\begin{array}{c}\frac{\widehat{X} \times \widehat{Z_{o^{\prime}}}}{\| \hat{X} \times \widehat{Z_{o^{\prime}} \|}}, \widehat{Y} \times \widehat{Z_{o^{\prime}}}=0 \\ \frac{\widehat{Y} \times \widehat{Z_{o^{\prime}}}}{\left\|\widehat{Y} \times \widehat{Z_{o^{\prime}}}\right\|}, \widehat{Y} \times \widehat{Z_{o^{\prime}} \neq 0}\end{array}, \widehat{Y_{o^{\prime}}}=\frac{\widehat{X} \times \widehat{Z_{o^{\prime}}}}{\| \widehat{X} \times \widehat{Z_{o^{\prime}} \|}}\right.$

We used fitting sphere to reconstruct boundary surface from cloud of boundary particle detected dynamically.
Fig. 8 Detection of boundary particle for a cylinder mold rotating around its main axis. The mold is shown in blue, the inner particle in green and boundary particle in red particles
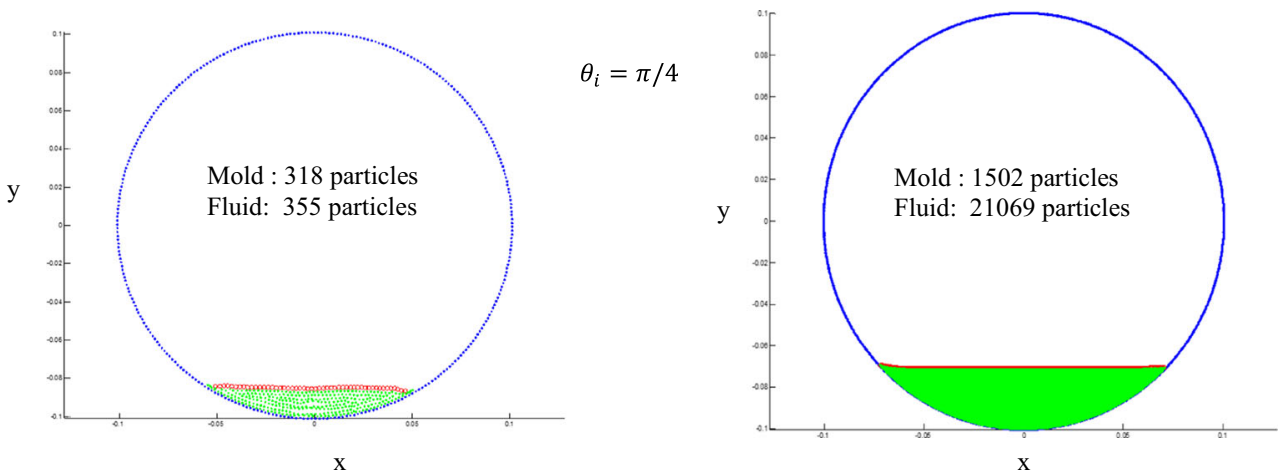
Fig. 9 Detection of boundary particle for a propeller mold rotating around its main axis at different moments. The mold is shown in blue, the inner particle in green and boundary particle in red particles

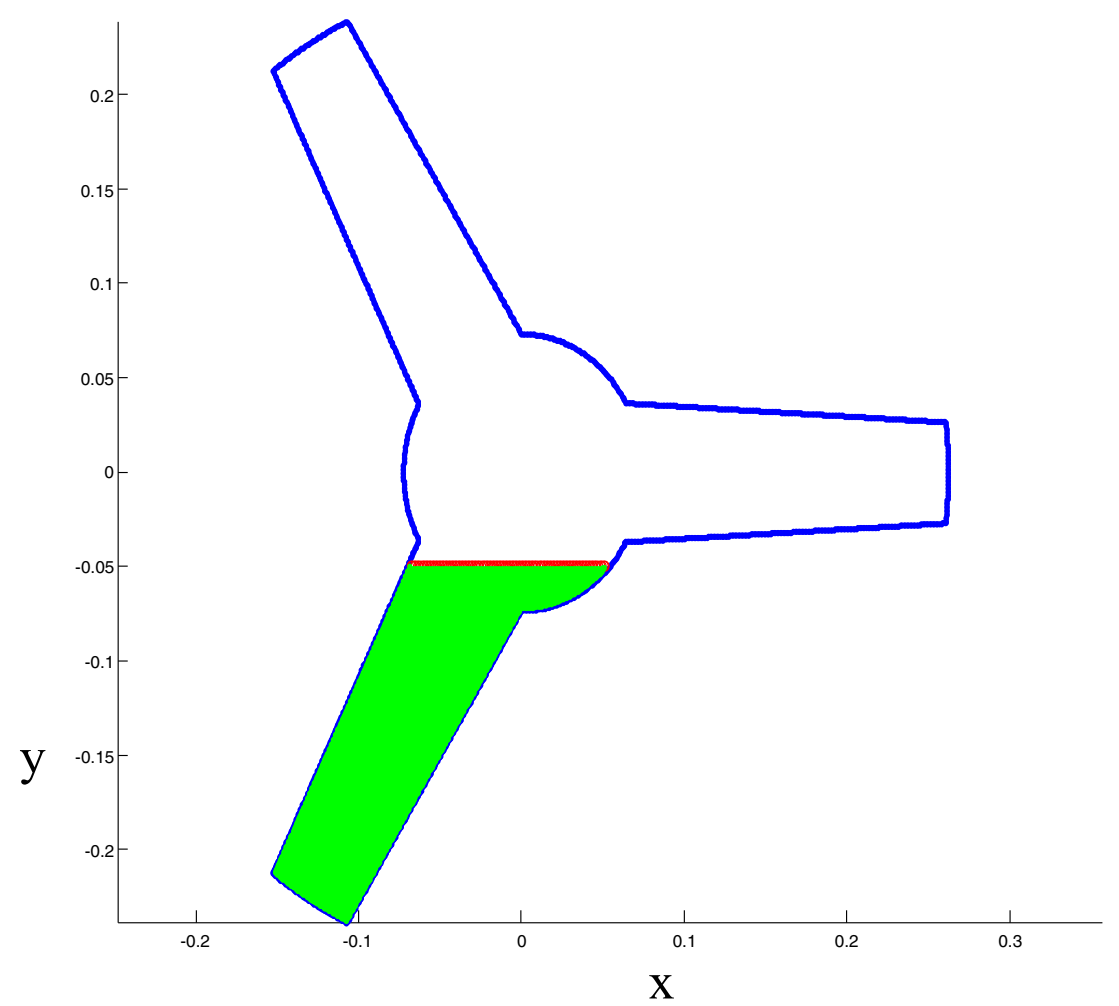

To fit sphere from data points, we used method developed by Ahn [34] based on least square.

The basic problem is to find a sphere that represents all data. With our sphere described by:

$(x-a)^{2}+(y-b)^{2}+(z-c)^{2}=R^{2}$

Where: $\mathrm{a}, \mathrm{b}, \mathrm{c}$ and $\mathrm{R}$ are the coordinate of the center and the radius of sphere.

This relation can be written as:

$x^{2}+y^{2}+z^{2}+x A_{1}+y A_{2}+z A_{3}+A_{0}=R^{2}$

Where

$A_{0}=a^{2}+b^{2}+c^{2}-R^{2} ; A_{1}=-2 a ; A_{2}=-2 b ; A_{3}=-2 c$

$$
\begin{aligned}
& {\left[\begin{array}{cccc}
n & \sum x_{k} & \sum y_{k} & \sum z_{k} \\
\sum x_{k} & \sum x_{k}^{2} & \sum x_{k} y_{k} & \sum x_{k} z_{k} \\
\sum y_{k} & \sum x_{k} y_{k} & \sum y_{k}^{2} & \sum y_{k} z_{k} \\
\sum z_{k} & \sum x_{k} z_{k} & \sum y_{k} z_{k} & \sum z_{k}^{2}
\end{array}\right]\left(\begin{array}{l}
A_{0} \\
A_{1} \\
A_{2} \\
A_{3}
\end{array}\right)} \\
& =-\left(\begin{array}{c}
\sum \rho_{k}^{2} \\
\sum \rho_{k}^{2} x_{k} \\
\sum \rho_{k}^{2} y_{k} \\
\sum \rho_{k}^{2} z_{k}
\end{array}\right)
\end{aligned}
$$

The resolution gives $A_{0}, A_{1}, A_{2}$ and the coordinate of sphere: $a=-\frac{A_{1}}{2} ; b=-\frac{A_{2}}{2} ; c=-\frac{A_{3}}{2}$; and the radius as:

$$
R=\sqrt{a^{2}+b^{2}+c^{2}-A_{0}}
$$

Fig. 10 Coalescence of two water droplets of at $25^{\circ} \mathrm{C}$ 
Fig. 11 Detection of boundary particle in three dimensional configurations. The mold is shown in blue, the inner particle in green and boundary particle in red colour

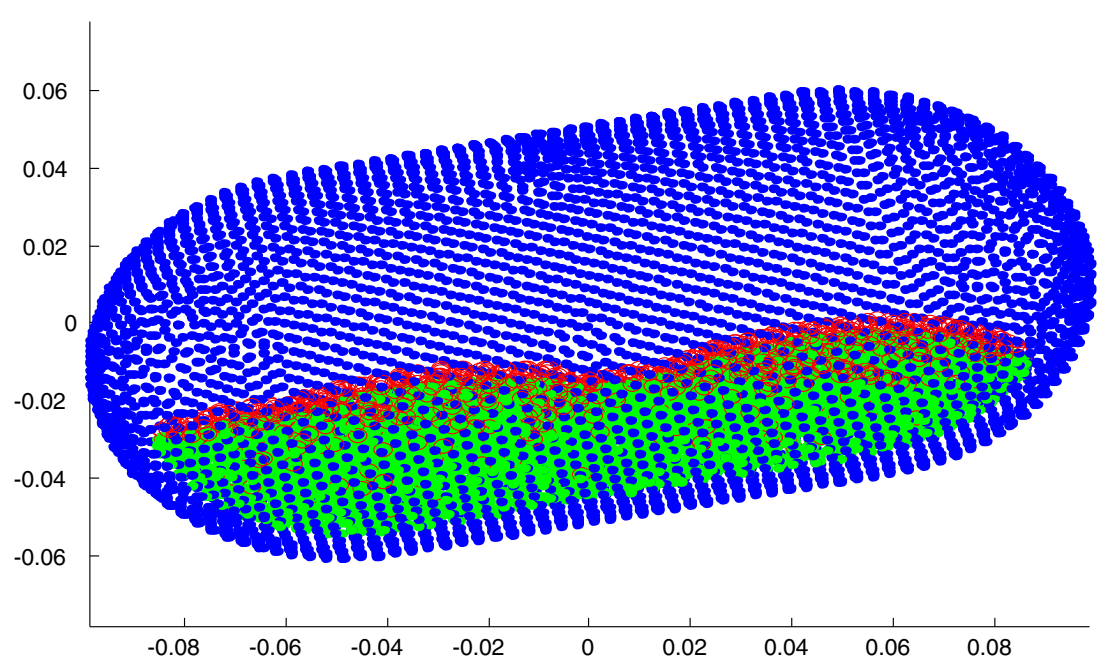

\section{Results and discussion}

Efficiency of detection boundary particle method

First, we used a mold with simple geometry; a cylinder rotating around its main axis (Fig. 8). The cylinder radius is $10 \mathrm{~cm}$ and the rotational speed is $7.5 \mathrm{rpm}(\mathrm{rpm})$. The mold is represented by 318 solid particles and its temperature is set at $60{ }^{\circ} \mathrm{C}$. The polymer which is polyurethane is represented by 355 of fluid particles, the initial temperature is set at $25^{\circ} \mathrm{C}$. The optimum angle $\theta_{i}=\pi / 4$, permit us to detect all the boundary particles. To test the efficiency of the scan cone, we increase the quantity of polymer in the mold (mold particle:
1502, fluid particle: 21069). The results obtained show that with same angle cone, the curve interface is perfectly detected which prove that the quantity of polymer didn't affect the value of scan angle.

To test the effect of geometry on the optimum angle scan value, we used propeller with three pales in $2 \mathrm{D}$ configuration (Fig. 9) where the mold is represented by 1574 mold particles and its temperature is set at $80^{\circ} \mathrm{C}$. The fluid is represented by 15,014 particles; the initial temperature is set at $25{ }^{\circ} \mathrm{C}$. With same angle $\theta \mathrm{i}=\pi / 4$, used for the simple geometry, we can find all the boundary particles.

In another part of this work, the ability of this method to detect the particles belonging to the border air-fluid was tested
Fig. 12 Particle position of 2D circle (radius $=0.1$ ) with the normal vector on the surface (obtained by the fitting circle and the Lagrangian interpolation)
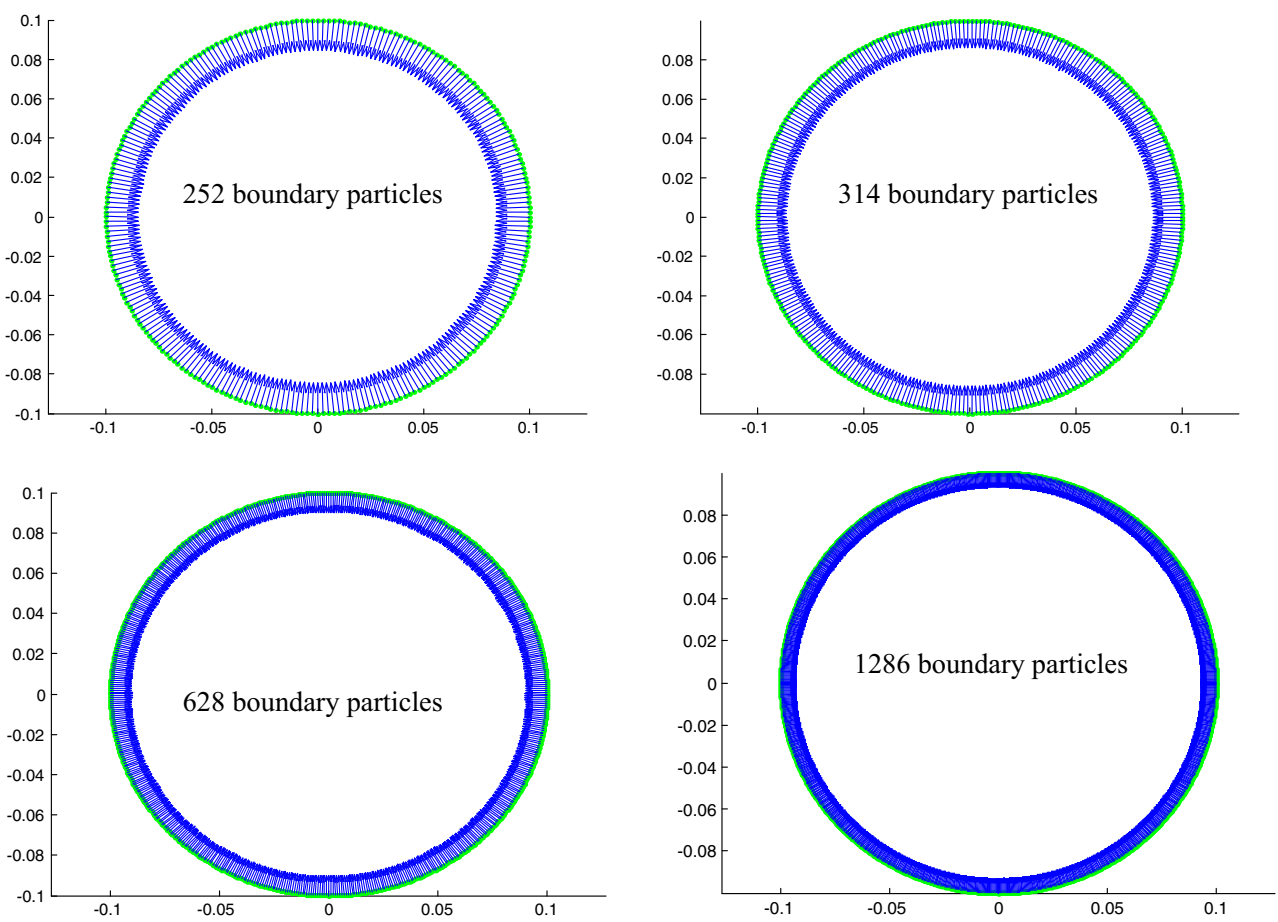
Fig. 13 Curvature of the reconstructed surface of $2 \mathrm{D}$ circle with radius of 0.1

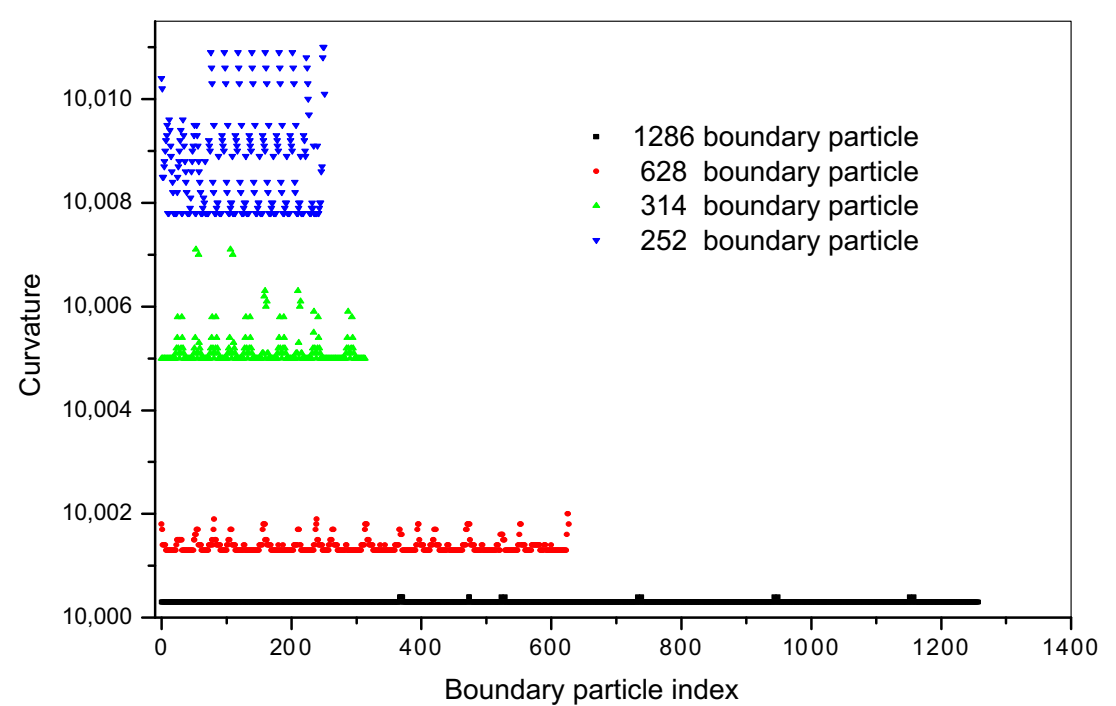

in the case of coalescence of two droplets of water in contact. The temperature was set at $25{ }^{\circ} \mathrm{C}$. The red particles represent boundary particles which detected with same angle (Fig. 10).

To test the efficiency of algorithm and to detect the surface boundary in three dimensional configurations, we used a cylinder as mold containing the reactive fluid and the scan cone angle $\theta_{\mathrm{i}}=\pi / 4$ (Fig. 11).

\section{Efficiency and accuracy of the surface tension model}

First, we performed our study on circle domain in order to show the capability of the two methods to estimate the normal and curvature of simple geometry like 2D circle (Fig. 12).

The both methods gave an accurate value of the normal. In the fitting circle method the value of curvature is constant and it's equal to ten which is the inverse of circle radius. On the other side, the value of curvature obtained by Lagrangian method is slight different. Because, in the circle method the curve passes over the set of boundary particles unlike in the second method the reconstructed curve didn't pass by the boundary particle which serves as origin of the interface curve. As shown in the Fig. 8 which describes the evolution of curvature in function of number particle boundary, the curvature values converge to ten by increasing the number of the boundary particles (Fig. 13). The relative errors for the 252, 314,628 and 1286 boundary particles are $0.084,0.051,0.013$ and $0.00301 \%$ respectively.

To validate the current method for surface tension, the computations of normal vector and curvature of the interface on sphere is studied. Figure 14 gives the calculated normal vector on the sphere.

The reconstruction surface method used in our study gives an accurate value of the normal and curvature. Indeed, the value of the curvature is constant and it's equal to ten which is the inverse of sphere radius.
Validation

We studied one benchmark, the two dimensional broken dam problems, to show the capability of our SPH solver to implement the effect of surface tension. This test is a classical benchmark problem for assessment of free surface flow and rapid motion [35]. The problem consists of a rectangular ( $\mathrm{H} \mathrm{x}$ L) column of fluid confined between a fixed wall and a temporary wall (dam). When, we removed the dam the fluid column start to collapse under the influence of gravity. We compared our results with those obtained experimentally by Martin and Moyce [36] for the collapse of a water column. In this simulation, the problem is represented by 4344 particles and the fluid viscosity is set at $0.001 \mathrm{~Pa}$.s (water at $20^{\circ} \mathrm{C}$ ).

In Fig. 15 the non-dimensional surge front positions of the collapsing dam $X^{*}=x / a$ are plotted against the non-

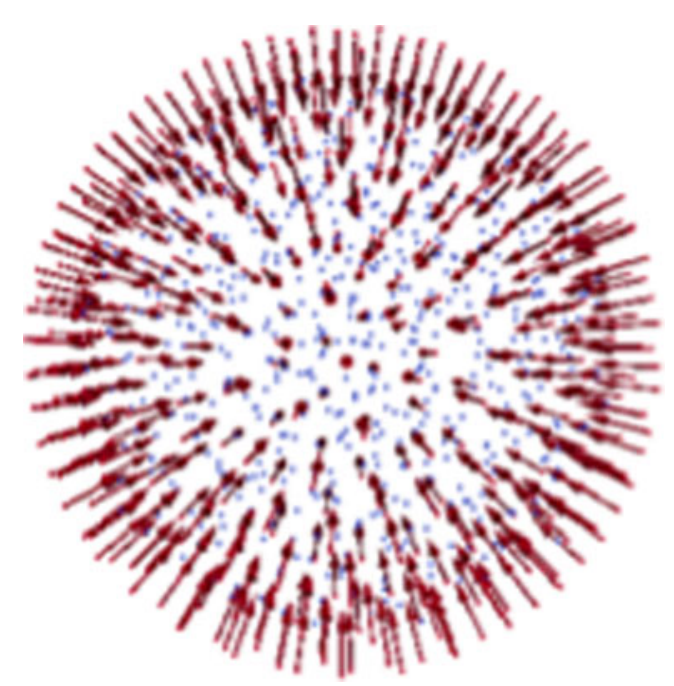

Fig. 14 Particle position of 3D sphere (radius $=0.1$ ) with the normal vector on the surface obtained by fitting sphere 
Fig. 15 Surge front for experiment $(\bullet)$ and $\mathrm{SPH}$ simulation ( $\mathbf{\square})$

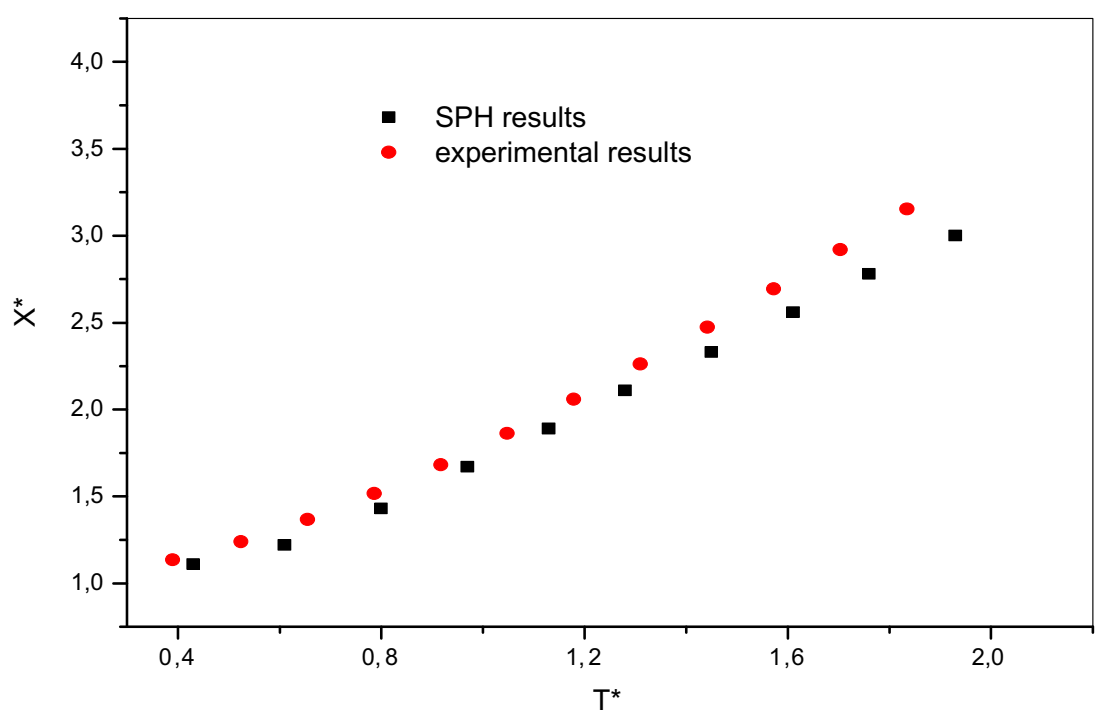

\section{Simulations applied to RRM}

$-2 \mathrm{D}$ simulations

We performed 2D simulations of an increasing viscosity fluid in a mold with or without taking into account the tension surface force, in order to show the effect of this force in our simulations. For this purpose, we used a simple geometry. The cylinder radius is $10 \mathrm{~cm}$ and the rotational speed is $7.5 \mathrm{rpm}$ (rpm).

The initial spacing particle is $1.10^{-3} \mathrm{~m}$. The mold is represented of approximately 636 solid particles and its temperature is set at $80^{\circ} \mathrm{C}$. The polymer which is a polyurethane is
Fig. 16 Shape of free surface at different periods of time

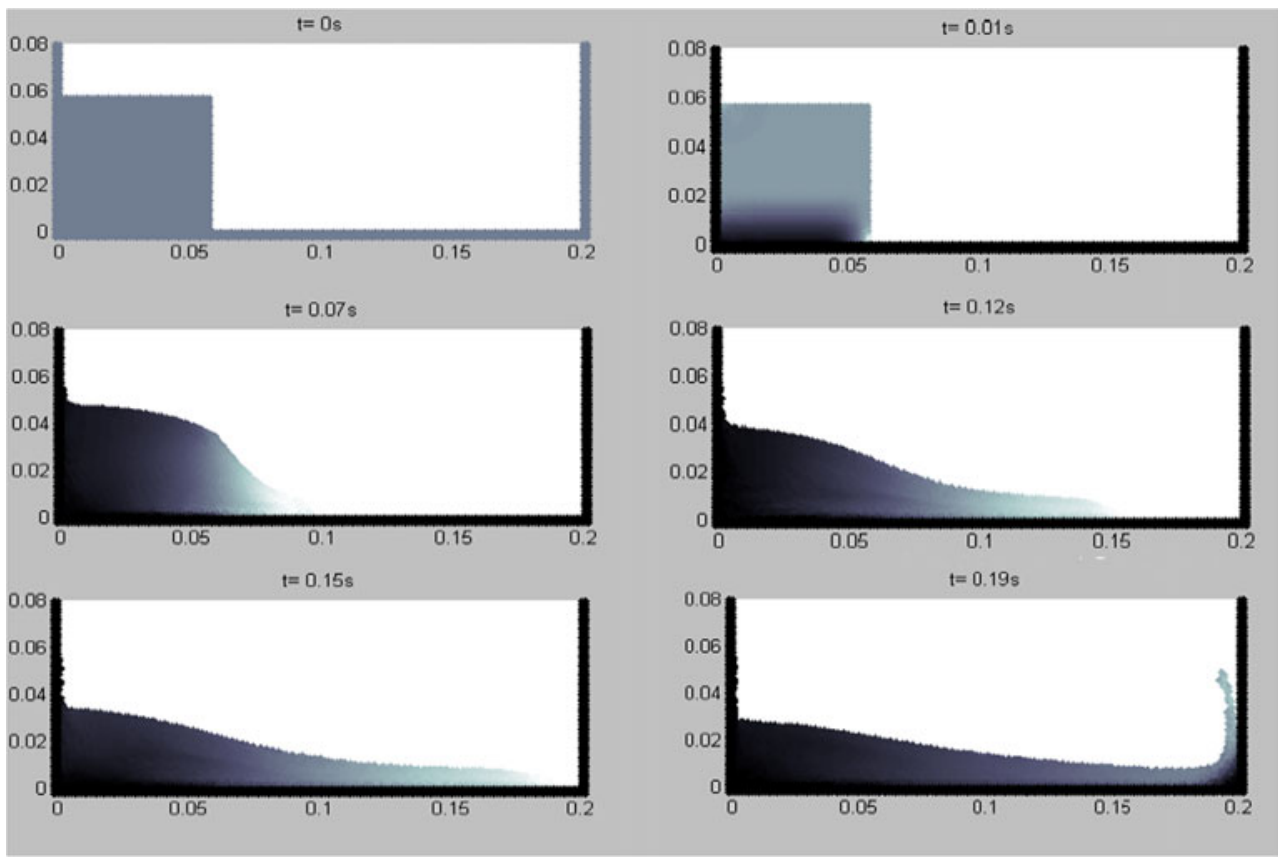




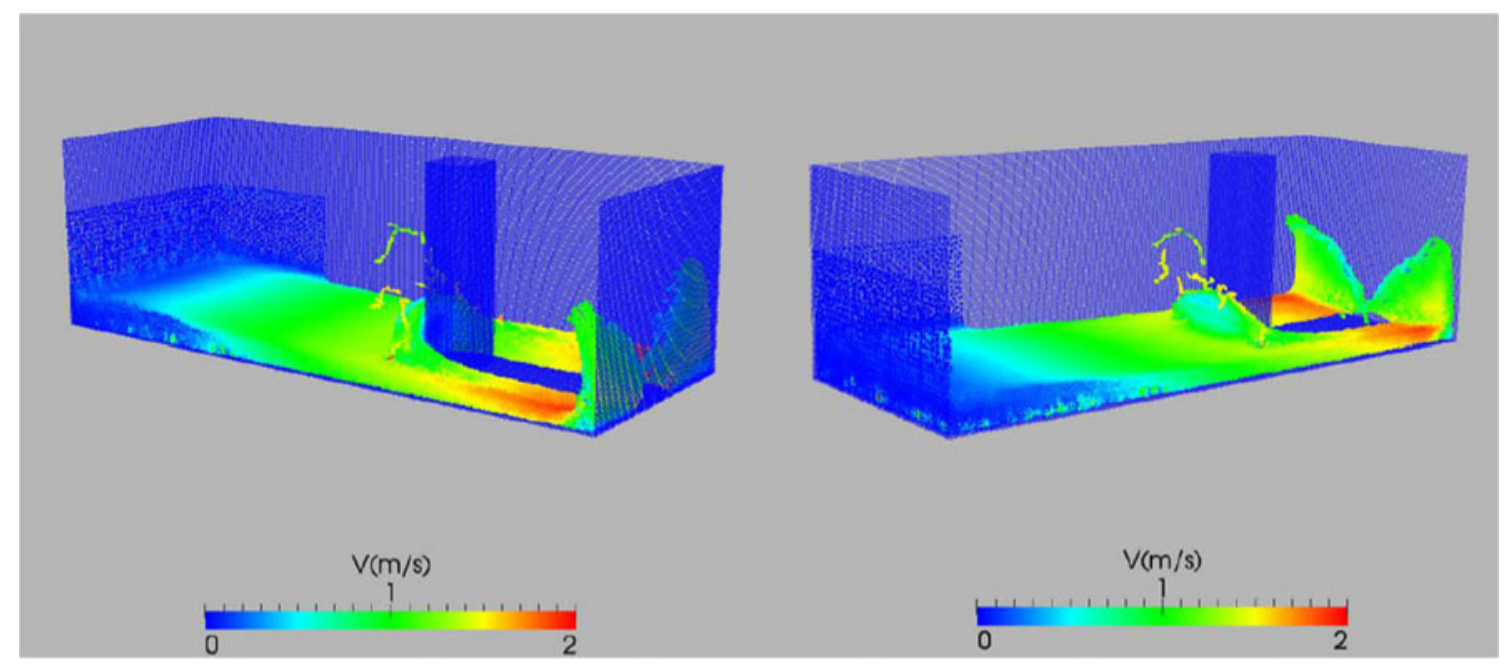

Fig. 17 3D dam break flow impacting with an obstacle

symbolized by 12387 fluid particles, the initial temperature is set at $25^{\circ} \mathrm{C}$. The parameters of adhesion models chosen are: $\eta_{\text {adhe }}=9$ Pa.s, $\mathrm{t}_{\text {adhe }}=3 \mathrm{~s}$ and $\Delta=1.5 * \mathrm{dx}$.

The coefficient of surface tension $\sigma$ is set to $30 \mathrm{mN} \cdot \mathrm{m}^{-1}$ (only for the simulation taking into account surface tension force).

The Fig. 18 represents the evolution of temperature of reactive fluid during RRM. At the beginning of the simulation, the reactive fluids have temperature equal to $25^{\circ} \mathrm{C}$ and there are no material fixed to the mould. After few seconds, the reaction starts and when the three conditions of adhesion model were satisfied, the polymer particles start to adhere to the internal surface (white particle). For heat transfer occurred between the mold and the polymer is governed by conduction; the temperature of polymer particle increased according to the Eq. (11) which we permit to observe gradient of temperature in the reactive system $(t=8,32 \mathrm{~s})$. we observe that the particle polymer near to the wall have temperature close to the mould whereas the internal fluid remains at his initial value which prove that the heat transfer is well presented.

In order to show the effect of surface tension, we undertake simulations with tension surface (in the left) and without this force (in the right). Figure 19 represents the simulations occurring with surface tension (on the left) and without this force (on the right). For the simulations with surface tension force, the internal surface shows less roughness than the simulation without taking into consideration the effect of surface tension. As we know, surface tension is caused by cohesive forces between the liquid's molecules. Inside the reactive fluid, each molecule is pushed and pulled equally in every direction by nearby molecules. The net force acting on these molecules is zero. The molecules at the surface however, do not have molecules on all sides. This results in a net force pointing inwards into the liquid. This creates internal pressure and forces the liquid's surface to contract to the smallest possible area which permits the decreasing of the curvature on the internal surface of the part.

To show the ability of our algorithm to simulate different kind of geometry, we used a mold of the rutland with three pales. The rotational speed was $7.5 \mathrm{rpm}$ (rpm).

The initial spacing particle was $8.10^{-4} \mathrm{~m}$. The mold was represented of approximately 1258 solid particles and its temperature is set at $80^{\circ} \mathrm{C}$. The mold was represented of approximately 1956 solid particles and its temperature is set at $80^{\circ} \mathrm{C}$. The polymer which was a polyurethane was symbolized of approximately 23000 fluid particles, the initial temperature was set at $25{ }^{\circ} \mathrm{C}$. With the initial amount of polymer, the final part wall thickness should be approximately of $5 \mathrm{~mm}$. At the end of the simulation, around ten particles should form the part thickness. The parameters of adhesion models chosen are: $\eta_{\text {adhe }}=9$ Pa.s, $\mathrm{t}_{\mathrm{adhe}}=3 \mathrm{~s}$ and $\Delta=1.5^{*} \mathrm{dx}$. The coefficient of surface tension $\sigma$ is set to $30 \mathrm{mN} \cdot \mathrm{m}^{-1}$.

Figure 20 shows the 2D simulations applied to RRM. When the three conditions of adhesion model are satisfied the reactive system start to fix to the mold. After $15 \mathrm{~s}$ we can observe a thin layer of polymer on the mold surface, represented by one or two particles. In the same time, heat transfer between the mold and the reactive material is well represented; the adhered particles are at the mold temperature whereas the fluid particles in the middle of the polymer are still at $25^{\circ} \mathrm{C}$. From $50 \mathrm{~s}$, tadhe starts to decrease significantly and combined to the increase of viscosity, fluid particles can quickly adhere to the surface of the mold. A polymer layer is formed of several particles.

\section{$-3 \mathrm{D}$ simulations}

Like to the two dimensional simulations, we conducted the numerical study with simple mold geometry; cylindrical mold 


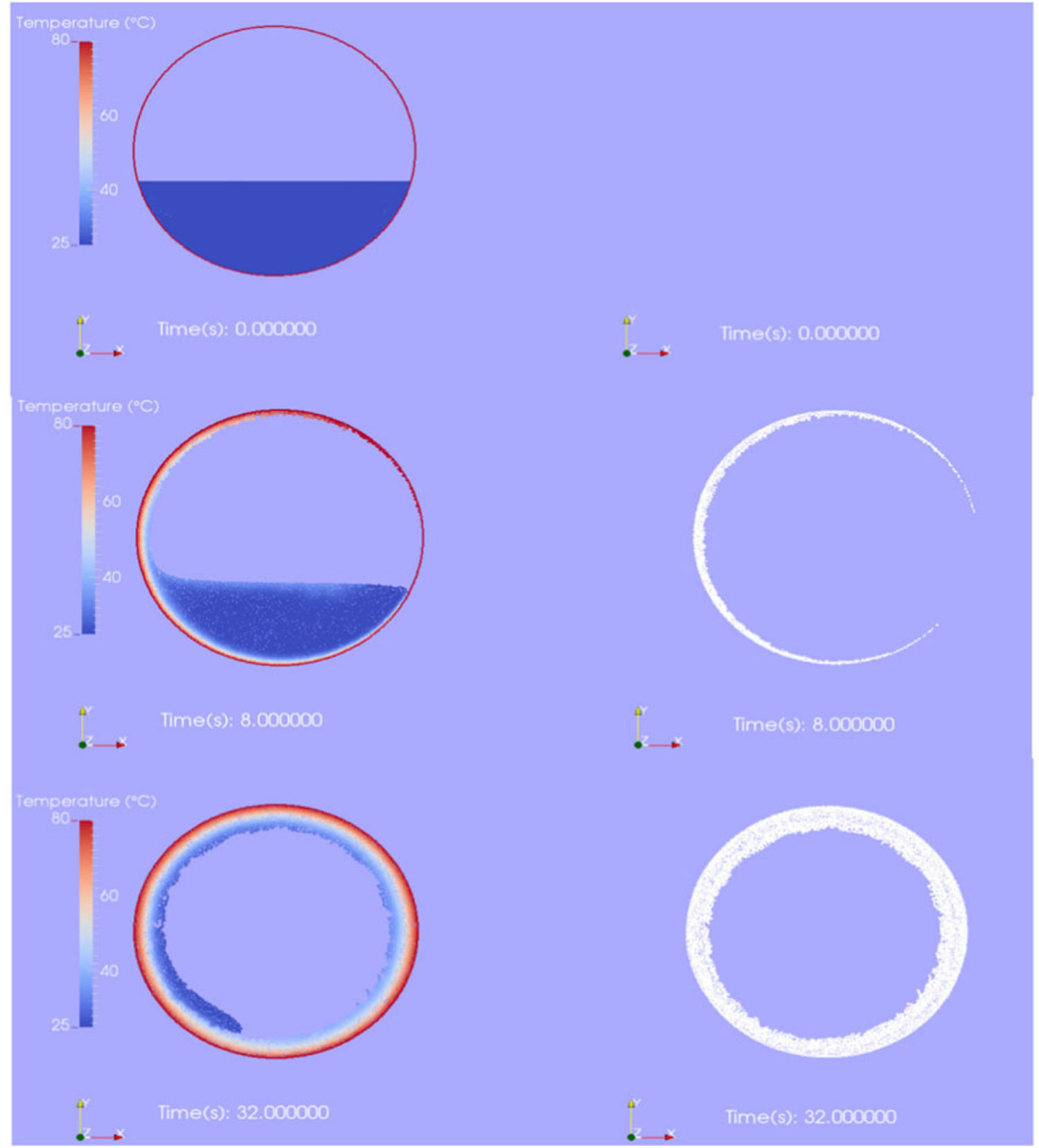

Fig. 18 Adhered polymer change and heat transfer during RRM at different periods (red, blue and white particles represent mold, reactive fluid and adhered polymer, respectively)

Fig. 19 The effect of surface tension force in the roughness of the internal surface of the part in the right: without tension surface, in the left: with tension force

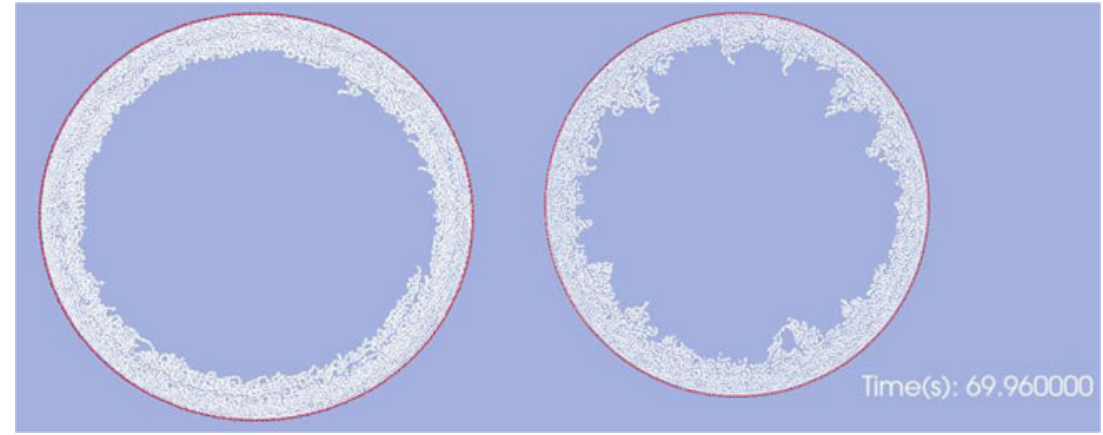


Fig. 20 Propeller with three pales

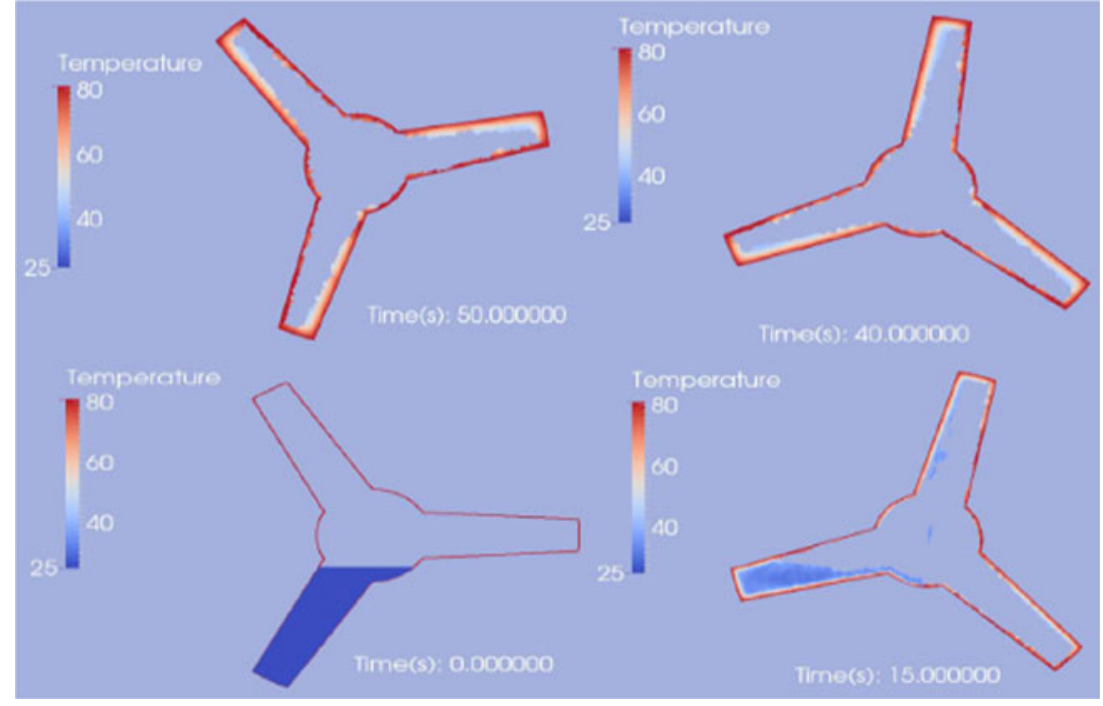

shape had a length of $20 \mathrm{~cm}$ and a diameter of $10 \mathrm{~cm}$. The parameters of adhesion models chosen were: $\eta_{\text {adhe }}=$ 30 Pa.s, $\mathrm{t}_{\mathrm{adhe}}=1.25 \mathrm{~s}$ and $\Delta=2 * \mathrm{dx}$. The coefficient of surface tension $\sigma$ was set to $30 \mathrm{mN} \cdot \mathrm{m}^{-1}$. The initial spacing particle was $2 \mathrm{~mm}$ which corresponds to $4 \mathrm{~mm}$ in final part. The mold was represented by 20 , 000 solid particles and its temperature is set at $80{ }^{\circ} \mathrm{C}$. The polymer which was polyurethane was represented by 90,000 of fluid particles; the initial temperature was set at $25{ }^{\circ} \mathrm{C}$ (Fig. 21). The evolution of viscosity follows the same law which was used in two dimensional configurations.

The fluid flow and heat transfer is shown in the Fig. 20. Once the three conditions of adhesion model are satisfied, the reactive starts to adhere in the internal surface of the mold.

Finally, we also simulated RRM for an industrial part, the inner part of a pressurized gas tank (Fig. 15). It consists of a liner surrounded by a polymer composite material. The inner liner should ensure water resistance and impermeability of the

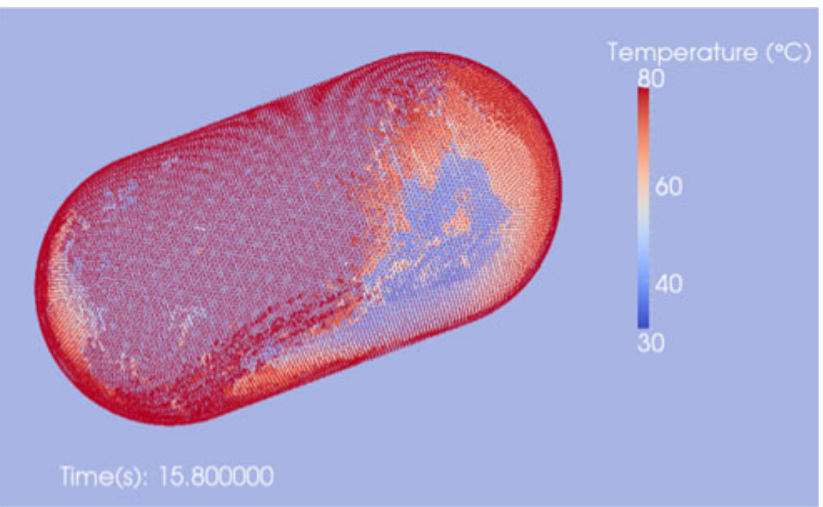

Fig. 21 3D cylinder (130,000 particles) tank while the composite material provides the mechanical strength. In the Fig. 21 (left), 120000 particles are used to simulate the shaping of the mold during RRM. The initial spacing fluid $\left(\mathrm{dx}=5 \cdot 10^{-3} \mathrm{~m}\right)$ and the velocities of two axes are set to 9 and $3 \mathrm{rpm}$, respectively.

With same adhesion model parameters used previously, we undertook this simulation. The SPH results describe the formation of the part and the evolutions of adhesion reactive system particle are shown in the Fig. 22.

At the beginning of the simulation, the reactive fluids have temperature equal to $25{ }^{\circ} \mathrm{C}$ and there are no material fixed to the mould. After few seconds, the reaction starts and once the three conditions of the adhesion model are satisfied, the reactive system starts to fix to the mold which are represented by white particles. Until $16 \mathrm{~s}$, we can observe the rimming flow, the material is not well distributed on the mould surface and the plastic part starts to appear and remaining fluid particles create a wave phenomenon on the fluid surface. At the end, we observe the formation of heterogeneous material at the internal surface of the mould and it's mainly concentrate at the corners of the liner. These results is very close to the reality where this phenomenon is observed when the axis rotates quickly which didn't give enough time to the material to fix homogenously. Therefore, the numerical results predict the real behaviour of reactive fluid during the process and it's necessary to reduce the velocity of the axis of rotation in order to obtain homogeneous piece.

Also, we note at this moment we can only perform simulations of few hundred thousands of particles, it takes already a long computational time. To do this, the solver should be parallelized on a graphical processing unit (GPU). However, the modeling of this kind of geometry proves the ability of our solver to treat complex geometry such as tank. 
Fig. 22 Numerical results of 3D SPH simulation of Liner: 120000 SPH particles (red, blue and white particles represent mold, reactive fluid and adhered polymer, respectively)

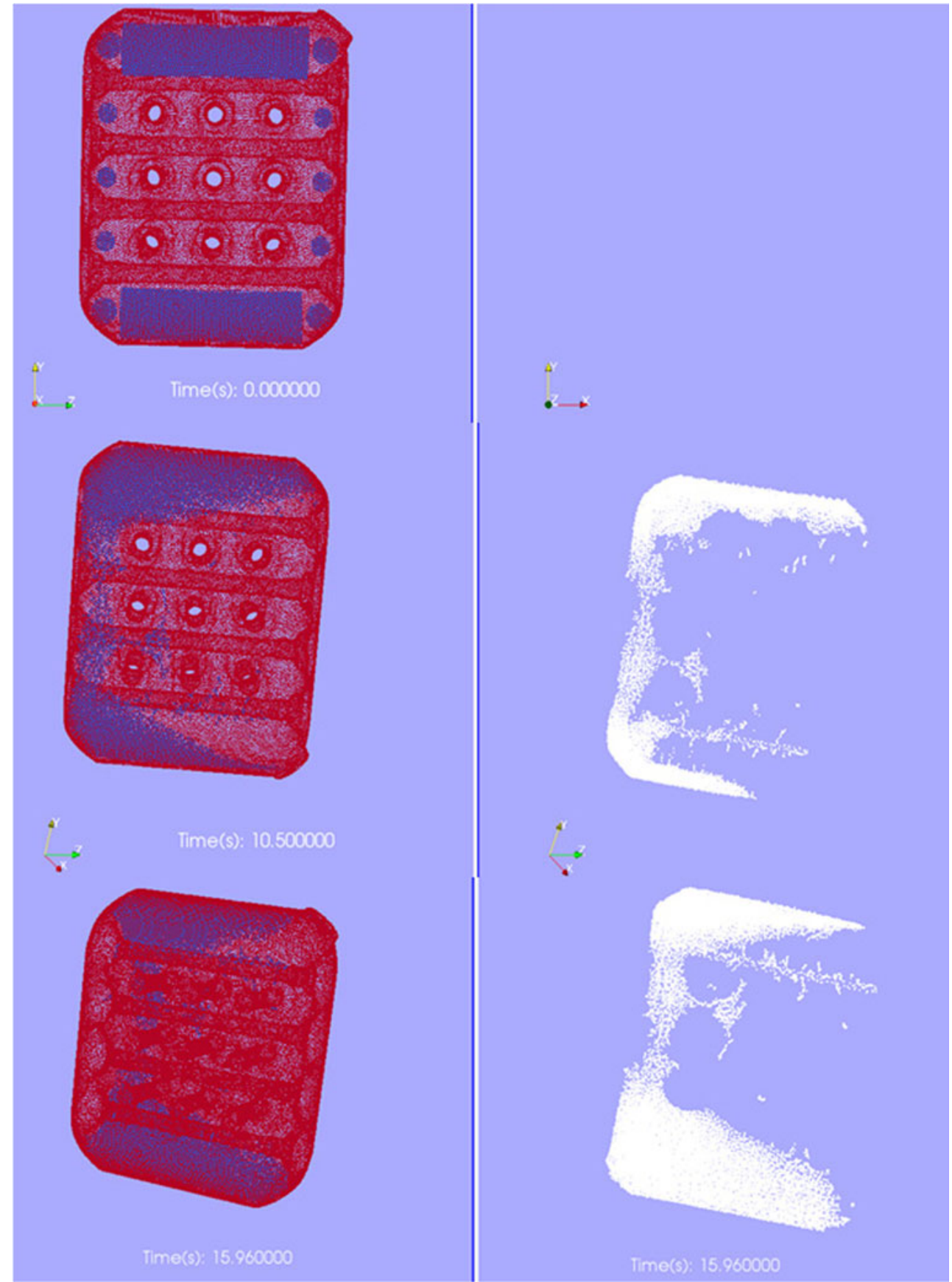

\section{Conclusion}

In this paper, an implementation of surface tension force in two and three dimensional SPH solvers were developed for simulation of polymer flow during RRM. Indeed, new rheokinetik model according to the operating conditions of shaping of parts is developed. The SPH method has been successfully applied to simulate Reactive Rotational Molding process in two and three SPH solvers. As we know, surface tension is important physical parameter and plays important role in the case of the final parts properties.
In $2 \mathrm{D}$, since the boundary particles are detected explicitly, the interface curve can be reconstructed by using two methods: Lagrangian interpolation or fitting circle and surface tension can be calculated directly on the surface. Surface tension force acts only on the surface which depends on the curvature. The both methods were validated by the estimation of normal and curvature of two dimension circles. This fitting circle method is simple to implement and we can avoid to calculate the derivative of the function which making it as an alternative method to the interpolation method. 
Also, in 3D the interface tracking is explicit and the surface can be reconstructed by using fitting sphere and surface tension can be calculated directly on the surface.

The method gives an accurate estimation and was validated in sphere domain and a represent an alternative method to Moving Least Square method which is widely used in SPH community. Additional, the method is a simple to implement rather than MLS method. Also, this method can be easily embedded in SPH solvers, without a significant increase of the CPU time.

The implementation of surface tension has improved the quality of our simulations by reducing the roughness of the internal surface. To enhance our solver, we will incorporate new criterion in the adhesion model and we also take account of the non-Newtonian character of polymer using viscoelastic models describing the behavior of our material during the process.

\section{References}

1. Barecasco A, Terissa H, Naa CF (2013) Simple free-surface detection in two and three-dimensional SPH solver

2. Terissa H, Barecasco A, Naa CF (2013) Three-dimensional smoothed particle hydrodynamics simulation for liquid droplet with surface tension

3. Tcharkhtchi A (2004) Rotomoulage de pièces en matière thermoplastique. Techniques de l'ingénieur Plastiques et composites (AM3706):AM3706. 3701-AM3715

4. Ortega Z, Monzon MD, Benitez AN, Kearns M, McCourt M, Hornsby PR (2013) Banana and abaca fiber-reinforced plastic composites obtained by rotational molding process. Math Manuf Proc 28(8):879-883

5. Rashmi BJ, Rusu D, Prashantha K, Lacrampe MF, Krawczak P (2013) Development of bio-based thermoplastic polyurethanes formulations using corn-derived chain extender for reactive rotational molding. Expr Polym Lett 7(10):852-862

6. Mounif E, Bellenger V, Ammar A, Ata R, Mazabraud P, Tcharkhtchi A (2008) Simulation de l'écoulement au cours du procédé de rotomoulage par la méthode Smoothed Particle Hydrodynamics (SPH). Mat Technol 96(6):263-268

7. Mounif E (2008) Résines époxy/amine pour le rotomoulage réactif: étude de la rhéocinétique et simulation numérique de l'écoulement. Thèse de doctorat, Arts et Métiers ParisTech

8. Riviere S, Khelladi S, Farzaneh S, Bakir F, Tcharkhtchi A (2013) Simulation of polymer flow using smoothed particle hydrodynamics method. Polym Eng Sci 53(12):2509-2518

9. Nugent S, Posch HA (2000) Liquid drops and surface tension with smoothed particle applied mechanics. Phys Rev E 62(4):4968-4975

10. Meleàn Y, Sigalotti LDG, Hasmy A (2004) On the SPH tensile instability in forming viscous liquid drops. Comp Phys Commun 157(3):191-200

11. Meleàn Y, Sigalotti LDG (2005) Coalescence of colliding van der Waals liquid drops. Int J Heat Mass Transf 48(19-20):4041-4061

12. Tartakovsky A, Meakin P (2005) Modeling of surface tension and contact angles with smoothed particle hydrodynamics. Phys Rev E 72(2):026301

13. Tartakovsky AM, Meakin P (2005) A smoothed particle hydrodynamics model for miscible flow in three-dimensional fractures and the twodimensional Rayleigh-Taylor instability. J Comp Physiol 207(2):610-624
14. Brackbill JU (1992) A continuum method for modeling surface tension. J Comp Physiol 100(2):335-354

15. Morris JP (2000) Simulating surface tension with smoothed particle hydrodynamics. Int J Numer Meth Fluids 33(3):333-353

16. Zhang M (2010) Simulation of surface tension in 2D and 3D with smoothed particle hydrodynamics method. J Comp Physiol 229(19): $7238-7259$

17. Dilts GA (2000) Moving least-squares particle hydrodynamics II: conservation and boundaries. Int J Numer Methods Eng 48(10): $1503-1524$

18. Haque A, Dilts GA (2007) Three-dimensional boundary detection for particle methods. J Comp Physiol 226(2):1710-1730

19. Marrone S, Colagrossi A, Le Touzé D, Graziani G (2010) Fast freesurface detection and level-set function definition in SPH solvers. J Comp Physiol 229(10):3652-3663

20. Randles PW, Libersky LD (1996) Smoothed particle hydrodynamics: some recent improvements and applications. Comp Meth Appl Mech Eng 139(1-4):375-408

21. Król P (1995) Generalization of kinetics in the reaction of isocyanates and polyols for modeling a process-yielding linear polyurethane, 1 . J Appl Polym Sci 57(6):739-749

22. Farzaneh S, Riviere S, Tcharkhtchi A (2012) Rheokinetic of polyurethane crosslinking time-temperature-transformation diagram for rotational molding. J Appl Polym Sci 125(2):1559-1566

23. Kamal M, Sourour S (1973) Kinetics and thermal characterization of thermoset cure. Pol Eng Sci 13(1):59-64

24. Castro J, Macosko C (1982) Studies of mold filling and curing in the reaction injection molding process. AIChE J 28(2):250-260

25. Pavier C, Gandini A (2000) Urethanes and polyurethanes from oxypropylated sugar beet pulp: I. Kinetic study in solution. Eur Pol J 36(8):1653-1658

26. Dong TW, Jiang SL, Huang XY, Liu HS, Huang QQ (2012) Investigation on the Non-Newtonian fluid flow in a single screw extruder using incompressible SPH (ISPH). Adv Mater Res 482: $745-748$

27. Violeau D (2012) Fluid mechanics and the SPH method theory and applications. Oxford University express, Oxford

28. Xu X, Ouyang J, Yang B, Liu Z (2013) SPH simulations of threedimensional non-Newtonian free surface flows. Comput Methods Appl Mech Eng 256:101-116

29. Takeda H, Miyama SM, Sekiya M (1994) Numerical simulation of viscous flow by smoothed particle hydrodynamics. Prog Theor Phys 92(5):939-960

30. Ata R, Soulaïmani A (2005) A stabilized SPH method for inviscid shallow water flows. Int J Numer Methods Eng 47(2):139-159

31. Roa MA, Argus MJ, Leidner D, Borst C, Hirzinger G (2012) Power grasp planning for anthropomorphic robot hands. In: Robotics and Automation (ICRA), 2012 I.E. Int Conf 14-18: 63-569

32. Pueschel P, Newnham G, Rock G, Udelhoven T, Werner W, Hill J (2013) The influence of scan mode and circle fitting on tree stem detection, stem diameter and volume extraction from terrestrial laser scans. ISPRS J Photogramm Remote Sens 77(5):44-56

33. Kasa I (1976) A circle fitting procedure and its error analysis. IEEE Trans Instrum Meas IM-25(1):8-14

34. Umbach D, Jones KN (2003) A few methods for fitting circles to data. IEEE Trans Instrum Meas 52(6):1881-1885

35. Ahn SJ, Rauh W, Warnecke H-J (2001) Least-squares orthogonal distances fitting of circle, sphere, ellipse, hyperbola, and parabola. Pattern Recogn 34(12):2283-2303

36. Violeau D, Issa R (2007) Numerical modelling of complex turbulent free-surface flows with the SPH method: an overview. Int J Numer Methods Eng 53(2):277-304

37. Martin JC, Moyce WJ (1952) Part IV. An experimental study of the collapse of liquid columns on a rigid horizontal plane. Philos Trans $\mathrm{R}$ Soc Lond A Math Phys Sci 244(882):312-324 